\title{
Evaluation of the Innovance D-DIMER analytical performance
}

\author{
Desiree Coen Herak, Marija Milos \\ and Renata Zadro*
}

Clinical Institute of Laboratory Diagnosis, Clinical Hospital Center Zagreb and University School of Medicine, Zagreb, Croatia

\begin{abstract}
Background: Widespread use of D-dimer in recent years has led to the development of a number of new fully automated quantitative D-dimer assays.

Methods: We evaluated the analytical performance of the particle-enhanced immunoturbidimetric assay Innovance D-DIMER (Siemens Medical Solutions) on the Behring Coagulation System (BCS) analyzer.

Results: Within-run coefficients of variation (CVs) for samples with low, borderline, slightly, and extremely increased D-dimer concentrations were $2.1 \%-5.5 \%$, whereas between-run CVs for control samples with low and extremely increased D-dimer were 5.5\%$8.4 \%$. The assay exhibited good linearity in the working range between $0.17 \mathrm{mg} / \mathrm{L}$ and $5.45 \mathrm{mg} / \mathrm{L}$ fibrinogen equivalent units (FEU), with the lower limit of detection of $0.099 \mathrm{mg} / \mathrm{L} \mathrm{FEU}$. The upper reference value determined in 40 plasma samples from healthy volunteers was $0.495 \mathrm{mg} / \mathrm{L} \mathrm{FEU}$. The results obtained in 457 fresh plasma samples were compared with results obtained with VIDAS D-Dimer Exclusion. Passing and Bablok regression analysis demonstrated highly significant correlation $(y=1.370 x-0.108$, $r=0.952, p<0.001)$. Bland and Altman difference plots demonstrated slightly higher results obtained with Innovance D-DIMER that was more pronounced with increasing values. Very good agreement between both assays was observed $(\kappa=0.860 ; 95 \%$ confidence interval (Cl), 0.811-0.908).
\end{abstract}

Conclusions: This study demonstrates that Innovance D-DIMER fulfills all analytical requirements for daily routine use.

Clin Chem Lab Med 2009;47:945-51.

Keywords: coagulation; D-dimer; evaluation; particleenhanced immunoturbidimetric assay.

\section{Introduction}

D-dimer is a stable terminal product of cross-linked fibrin generated by plasmin degradation. As D-dimer antigen is a specific indicator of fibrin formation, it is

\footnotetext{
*Corresponding author: Renata Zadro, Clinical Institute of Laboratory Diagnosis, Zagreb University School of Medicine and Clinical Hospital Center, Zagreb, Croatia Phone: + 38512367360, Fax: +38512367395

E-mail: rzadro@mef.hr

Received February 23, 2009; accepted May 7, 2009; previously published online June 24, 2009
}

currently the most widely used laboratory marker for in vivo coagulation activation (1).

Measurement of D-dimer concentrations has gained widespread use in the diagnosis of various clinical situations related to thrombosis and fibrinolysis. It is especially useful as a diagnostic tool for excluding suspected thromboembolic disease. Thus, coagulation laboratories are faced with an increasing number of requests for the determination of $D$-dimer.

Currently, more than 30 assays for the determination of D-dimer antigen are commercially available. These assays are based on more than 20 different D-dimer specific monoclonal antibodies that react with conformational epitopes generated by factor XIlla-induced cross-linking on fibrin fragment D-dimer, that are not present on fibrinogen fragment $D$, other fibrinogen degradation products or native fibrinogen (2). Several manufacturers have recently produced a variety of new fully automated quantitative D-dimer assays that are simple to perform and suitable for individual and rapid testing. The majority of these assays are latex-enhanced photometric immunoassays (LPIA) developed for the measurement of Ddimer in plasma samples on coagulation analyzers, that can be performed simultaneously with other routine coagulation assays.

The aim of this study was to evaluate the analytical performance of the recently developed particleenhanced immunoturbidimetric assay Innovance DDIMER on the Behring Coagulation System (BCS) analyzer (Siemens Medical Solutions Diagnostics, Deerfield, IL, USA).

\section{Materials and methods}

\section{Principle of the D-dimer measurement}

Innovance D-DIMER (Siemens Medical Solutions Diagnostics, Deerfield, IL, USA) is a fully automated particleenhanced immunoturbidimetric assay for the quantitative determination of D-dimer in plasma. The assay relies on the 8D3 monoclonal antibody, covalently coupled to polystyrene particles, and is designed for performance on several automated coagulation analyzers from Siemens Medical Solutions Diagnostics.

In this study, D-dimer testing was performed using the BCS coagulation analyzer (Siemens Medical Solutions Diagnostics) with the Innovance D-DIMER Kit. The kit consists of D-DIMER Reagent, D-DIMER Buffer, D-DIMER Supplement, D-DIMER Diluent and D-DIMER Calibrator, according to the manufacturer's specifications. To a mixture of $9 \mu \mathrm{L}$ of $\mathrm{D}$ DIMER Diluent and $9 \mu \mathrm{L}$ of plasma, $72 \mu \mathrm{L}$ of D-DIMER Buffer and $27 \mu \mathrm{L}$ of D-DIMER Supplement were added and incubated at $37^{\circ} \mathrm{C}$ for $180 \mathrm{~s}$. The degree of agglutination was measured after the addition of $72 \mu \mathrm{L}$ of D-DIMER Reagent (suspension of polystyrene particles covalently coated with a monoclonal mouse antibody 8D3) as the decrease of transmitted light at $570 \mathrm{~nm}$ caused by aggregates. The degree of 
agglutination was directly proportional to the concentration of D-dimer in the sample and results were expressed in $\mathrm{mg} / \mathrm{L}$ fibrinogen equivalent units (FEU).

The measuring range of the assay is defined by the lotspecific concentration of D-DIMER calibrator included in the reagent kit. Concentrations range from $\sim 0.19 \mathrm{mg} / \mathrm{L}$ to $4.40 \mathrm{mg} / \mathrm{L} \mathrm{FEU}$, which can be extended to $\sim 35.20 \mathrm{mg} / \mathrm{L} \mathrm{FEU}$ by automatic redilution (1:8) of samples with results above $4.40 \mathrm{mg} / \mathrm{L} \mathrm{FEU}$. In addition to lot-specific concentrations, each calibrator has a declared analyzer specific concentration of D-dimer.

In this study, a six-point calibration was performed automatically by the instrument using the D-DIMER Calibrator with a D-dimer concentration of $5.54 \mathrm{mg} / \mathrm{L} \mathrm{FEU} \mathrm{declared} \mathrm{for}$ BCS/BCS XP analyzers. Two calibration curves were prepared: a lower one spanning $0.17-4.26 \mathrm{mg} / \mathrm{L} \mathrm{FEU}$, and a higher one up to $34.09 \mathrm{mg} / \mathrm{L} \mathrm{FEU.}$

\section{Evaluation samples}

Two types of samples were used for the evaluation procedure: consecutive patient samples submitted to the Clinical Institute for Laboratory Diagnosis of the Clinical Hospital Center Zagreb, Croatia for the determination of D-dimer, and samples obtained from apparently healthy volunteers who were members of the hospital staff.

Platelet-poor plasma was prepared by centrifugation at $2000 \mathrm{~g}$ for $15 \mathrm{~min}$ at room temperature of blood specimens collected into siliconized glass tubes containing $0.105 \mathrm{~mol} / \mathrm{L}$ buffered sodium citrate (Becton Dickinson, Plymouth, UK) in a ratio of nine parts of blood to one part anticoagulant. Fresh plasma samples obtained within $2 \mathrm{~h}$ following collection were used for the determination of the assay specific reference interval, precision and method comparison study. Commercially available lyophilized plasma samples Innovance D-DIMER Control: D-DIMER Control 1 (C1) for assessing the lower measurement range, and D-DIMER Control 2 (C2) for assessing the upper measurement range were used for quality control and for precision studies.

Four types of samples were used in the method comparison study: samples from healthy controls, plasma samples from patients with a wide range of D-dimer concentrations, lyophilized control samples (Innovance D-DIMER C1 and C2 from Siemens; VIDAS C1 and VIDAS C2 from bioMérieux, Marcy I'Étoile, France), calibrators (Innovance D-DIMER Calibrator from Siemens and VIDAS S1, VIDAS S2 from bioMérieux), and six lyophilized citrated plasma samples kindly supplied by the UK National External Quality Assessment Scheme (UK NEQAS) for blood coagulation.

\section{Evaluation procedure}

The evaluation procedure included determination of withinrun and between-run precision, accuracy, linearity and lower limit of detection, verification of the assay-specific reference interval and method comparison studies.

Within-run precision was determined using three fresh plasma samples with different D-dimer concentrations: sample 1 (low D-dimer), sample 2 (borderline D-dimer) and sample 3 (slightly increased D-dimer), and control plasma sample Innovance D-DIMER C2 (extremely increased Ddimer). For each sample, 20 replicates were analyzed in the single analytical run.

Between-run precision was assessed using two control plasma samples (Innovance D-DIMER C1 and C2). Samples were analyzed over 20 consecutive days with duplicate determinations performed in the morning (samples A) and in the afternoon (samples B) on each day. Within- and between-run precision was determined using the same calibration curve.
Accuracy was evaluated by calculating the bias from the target value for control plasma samples.

Linearity was evaluated by analysis of multiple dilutions of a patient sample with increased D-dimer concentrations ( $5.45 \mathrm{mg} / \mathrm{L}$ FEU) using the original calibration curve. Seven dilutions were prepared using Innovance D-DIMER Diluent at fixed ratios of $4: 5,3: 4,1: 2,1: 4,1: 8,1: 16$ and 1:32. Each dilution was assayed in duplicate and percent recovery was calculated from the measured mean values for each dilution.

The lower limit of detection of the assay was determined by measurement of D-dimer concentrations in a sample without D-dimer (Innovance D-DIMER DILUENT) and calculated as the mean value +3 SD (3).

Assay-specific reference intervals were verified by measurement of D-dimer concentrations in citrated blood samples obtained from 40 apparently healthy volunteers (members of the hospital staff) who had no known defects of blood coagulation.

For method comparison studies, samples were tested in parallel with Innovance D-DIMER vs. the routine assay normally used in our laboratory: the VIDAS D-Dimer Exclusion on the mini VIDAS Immunoassay system (bioMérieux, Marcy I'Étoile, France). The principle of the VIDAS D-Dimer Exclusion assay utilizes a two-step enzyme immunoassay sandwich method using two different monoclonal antibodies (10B5E12C9 and 2C5A10) and fluorescent detection (4). Results are reported in $\mathrm{mg} / \mathrm{L}$ FEU, with a measurement range from $0.05 \mathrm{mg} / \mathrm{L}$ to $10.0 \mathrm{mg} / \mathrm{L}$ FEU. Both D-dimer methods were performed according to the manufacturer's recommendations and the same cut-off value $(<0.5 \mathrm{mg} / \mathrm{L}$ FEU) was used for both assays.

\section{Statistical analysis}

The MedCalc program version 9.3.2.0 for Windows (MedCalc Software, Mariakerke, Belgium) was used for statistical analysis. Correlation between methods was calculated according to Spearman and regression analysis was performed using the method of Passing and Bablok (5). The 95\% confidence intervals (Cls) were calculated according to binomial distribution. The agreement between results was evaluated according to Bland and Altman (6). Overall agreement between both assays in their diagnostic classification was estimated by calculation of the kappa coefficient (к) for agreement (7), and the obtained value was evaluated according to Landis and Koch (8).

\section{Results}

\section{Within-run and between-run precision}

Results of within-run and between-run precision studies are shown in Table 1. The within-run coefficients of variation (CVs) ranged from $2.1 \%$ for the control sample with extremely increased D-dimer concentrations to $5.5 \%$ for the patient sample with low D-dimer concentrations. The between-run $\mathrm{CV}$ s ranged from $5.5 \%$ to $8.4 \%$, with slightly higher CVs obtained in the morning run compared to CVs obtained in the afternoon run, for both control samples.

For the study on accuracy, the bias from the target values for the commercial control samples, Innovance D-DIMER C1 and C2, were $7.7 \%$ and 7.3\%, respectively. 
Table 1 Within-run and between-run precision of the Innovance D-DIMER assay.

\begin{tabular}{|c|c|c|c|c|}
\hline & $\mathrm{n}$ & $\begin{array}{l}\text { Mean } \pm S D \\
\mathrm{mg} / \mathrm{L} \text { FEU }\end{array}$ & $\begin{array}{l}\text { Range, } \\
\text { mg/L FEU }\end{array}$ & $\mathrm{CV}, \%$ \\
\hline \multicolumn{5}{|l|}{ Within-run } \\
\hline Sample 1 & 20 & $0.31 \pm 0.02$ & $0.29-0.35$ & 5.5 \\
\hline Sample 2 & 20 & $0.50 \pm 0.01$ & $0.47-0.52$ & 2.6 \\
\hline Sample 3 & 20 & $0.81 \pm 0.04$ & $0.71-0.85$ & 4.5 \\
\hline Innovance D-DIMER Control 2 & 20 & $3.02 \pm 0.06$ & $2.89-3.10$ & 2.1 \\
\hline \multicolumn{5}{|l|}{ Between-run* } \\
\hline \multicolumn{5}{|l|}{ Innovance D-DIMER Control 1} \\
\hline A & 20 & $0.37 \pm 0.03$ & $0.33-0.45$ & 8.4 \\
\hline B & 20 & $0.35 \pm 0.02$ & $0.32-0.41$ & 7.1 \\
\hline \multicolumn{5}{|l|}{ Innovance D-DIMER Control 2} \\
\hline A & 20 & $3.18 \pm 0.20$ & $2.78-3.43$ & 6.2 \\
\hline B & 20 & $3.00 \pm 0.17$ & $2.68-3.25$ & 5.5 \\
\hline
\end{tabular}

*Innovance D-DIMER Control 1 and 2 were measured in the morning (run A) and in the afternoon (run B) of each day. SD, standard deviation; FEU, fibrinogen equivalent units; CV, coefficient of variation.

\section{Linearity and lower limit of detection}

The results obtained for the linearity study are shown in Table 2. The mean recovery was $99.1 \%$, ranging from $88.2 \%$ to $108.8 \%$. The correlation coefficient for linear regression analysis comparing measured and expected D-dimer concentrations was $r^{2}=0.995$, with a regression line $y=0.949 x+0.068$. These results indicate that the assay exhibited very good linearity in the working range of $0.17-5.45 \mathrm{mg} / \mathrm{L} \mathrm{FEU}$, which is even greater than the measuring range stated from the manufacturer.

The lower limit of detection was $0.099 \mathrm{mg} / \mathrm{L}$ FEU.

\section{Reference interval}

The median D-dimer concentration was $0.27 \mathrm{mg} / \mathrm{L}$ FEU and the highest measured concentration was $0.55 \mathrm{mg} / \mathrm{L}$ FEU. The upper reference limit based on the 95 th percentile was $0.495 \mathrm{mg} / \mathrm{L}$ FEU.

\section{Method comparison}

A total of 457 fresh plasma samples (40 plasma samples from healthy controls and 417 plasma samples from inpatients and outpatients) were analyzed using both assays. For statistical analysis, 38 samples with results outside the measurement range were excluded.

Linear regression analysis according to Passing and Bablok (Figure 1) showed significant correlation $(p<0.001)$. Better correlation $(r=0.930)$ was obtained for samples with values up to $4.26 \mathrm{mg} / \mathrm{L}$ FEU (undiluted samples), compared to a correlation coefficient of 0.748 for samples with concentrations $>4.26 \mathrm{mg} / \mathrm{L}$ FEU, and obtained following automatic dilution.

The results obtained with both assays were also evaluated according to the method of Bland and Altman. The analysis included absolute difference plots, percent difference plots and ratio difference plots. These analyses were performed for all samples, and separately for undiluted, and diluted samples. Percent difference plots and ratio difference plots are shown in Figure 2. Mean absolute difference between Innovance D-DIMER and VIDAS D-Dimer Exclusion was $0.8 \mathrm{mg} / \mathrm{L} \mathrm{FEU} \mathrm{for} \mathrm{all} \mathrm{samples} \mathrm{that} \mathrm{were} \mathrm{tested,} 0.2$ $\mathrm{mg} / \mathrm{L}$ FEU for undiluted samples and $4.8 \mathrm{mg} / \mathrm{L}$ FEU for diluted samples.

Agreement between methods, defined as the percentage of values found concomitantly positive or negative between Innovance D-DIMER and VIDAS DDimer Exclusion, was $94.3 \%$. Concordance between results was observed in $96.5 \%$ of samples with results above the cut-off value and in $97.8 \%$ of samples below the cut-off value. If we assumed the Vidas to be the gold standard, sensitivity was $96.1 \%$, specificity $89.1 \%$, negative predictive value $93.4 \%$, and the positive predictive value $93.5 \%$. The obtained $\kappa$ was 0.860 (95\% Cl, 0.811-0.908).

The results obtained for commercial D-dimer control samples and calibration plasma samples from

Table 2 Determination of linearity of the Innovance D-DIMER assay on the BCS analyzer using multiple dilutions of a patient sample with increased D-Dimer concentrations.

\begin{tabular}{|c|c|c|c|c|c|}
\hline \multicolumn{2}{|c|}{ Dilution } & \multicolumn{2}{|c|}{ Measured mean value } & \multirow{2}{*}{$\begin{array}{l}\text { Expected value, } \\
\mathrm{mg} / \mathrm{L} \text { FEU }\end{array}$} & \multirow{2}{*}{$\begin{array}{l}\text { Recovery, } \\
\%\end{array}$} \\
\hline Ratio & $\%$ & $\mathrm{mE} / \mathrm{min}$ & $\mathrm{mg} / \mathrm{L} \mathrm{FEU}$ & & \\
\hline $1: 1$ & 100 & 577.7 & 4.99 & 5.45 & 91.6 \\
\hline 4:5 & 80 & 507.0 & 4.29 & 4.36 & 98.4 \\
\hline $3: 4$ & 75 & 493.3 & 4.17 & 4.09 & 102.0 \\
\hline $1: 2$ & 50 & 338.7 & 2.86 & 2.72 & 105.1 \\
\hline $1: 4$ & 25 & 145.7 & 1.42 & 1.36 & 104.4 \\
\hline $1: 8$ & 12.5 & 67.79 & 0.74 & 0.68 & 108.8 \\
\hline 1:16 & 6.25 & 32.16 & 0.32 & 0.34 & 94.1 \\
\hline 1:32 & 3.125 & 19.98 & 0.15 & 0.17 & 88.2 \\
\hline
\end{tabular}

FEU, fibrinogen equivalent units. 
A

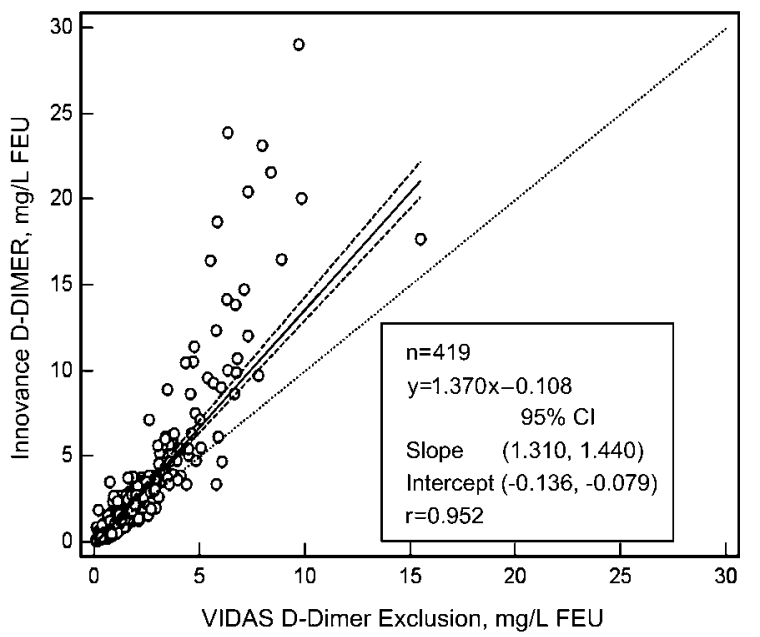

B

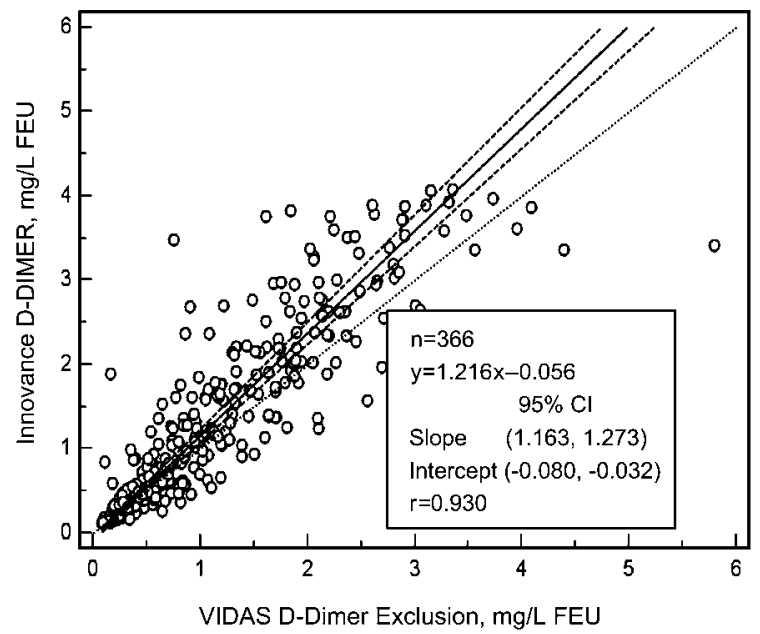

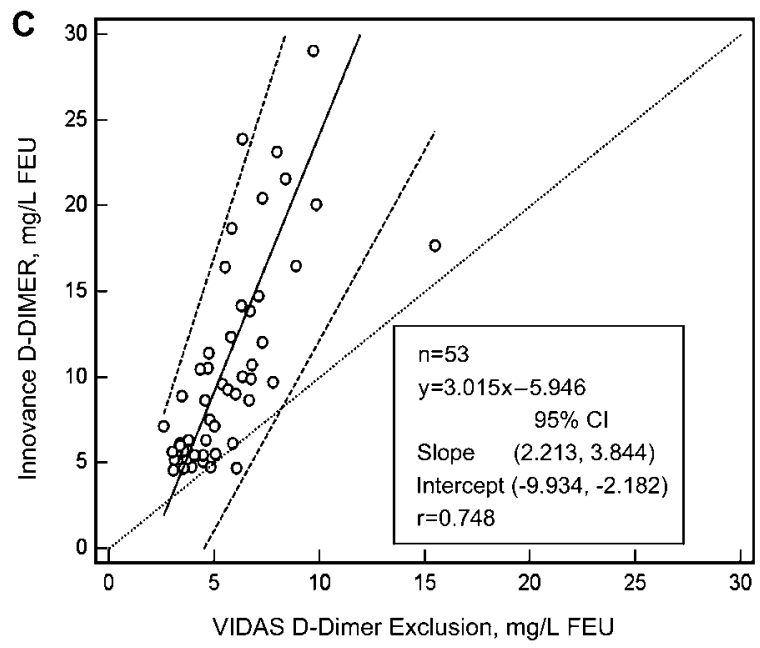

Figure 1 Method comparison between Innovance D-DIMER and VIDAS D-Dimer Exclusion according to Passing and Bablok. All tested samples (A), undiluted samples (B) and diluted samples (C).

both manufacturers are shown in Table 3. Values were $\sim 3$-fold higher for VIDAS control samples and calibrators measured with the Innovance D-DIMER method.

Method comparison results for UK NEQAS plasma samples are summarized in Table 4 . Increased values were observed for all samples with Innovance DDIMER. Ratios ranged from 1.22 to 1.51 , except for one sample where similar D-dimer concentrations were measured with both assays.

\section{Discussion}

In recent years, a growing number of quantitative Ddimer assays have been developed for use in routine laboratory practice. The majority of these assays are fully automated LPIA that can be performed using different coagulation analyzers and simultaneously with other routine coagulation assays. Every new assay needs to be validated in terms of its analytical and clinical performance prior to implementation for daily use. Thus, the data from this study summarize the analytical performance of the Innovance D-DIMER assay on the BCS coagulation analyzer.
Innovance D-DIMER shares the same characteristics of other LPIA D-dimer assays. The method is fully automated, uses a small amount of sample per test $(9 \mu L)$ and has a short sample turnaround time of $10 \mathrm{~min}$. The assay displayed very good linearity, up to $5.45 \mathrm{mg} / \mathrm{L} \mathrm{FEU}$, that was greater than the measurement range stated by the manufacturer. A possible reason for this could be differences in the composition of the mixture of D-dimer-containing fragments in the calibrator and samples used for the assessment of linearity $(1,9)$, although the calibrator and samples were both of human origin. The wide linear range offers the advantage of direct measurement of the majority of samples $(88.2 \%)$, without the need for dilution and thus decreased turnaround time. Although the wide measurement range provides several benefits, one limitation is the usually high CV at low concentrations (9), which was reproducible in the present study. However, assay reproducibility at concentrations near the cut-off value that represent the critical point of the assay, as stressed by Reber and de Moerloose (9), was very low ( $C V=2.6 \%$ ).

The lower limit of detection of the assay was almost two-fold lower than the lowest calibrator value (0.099 mg/L FEU). We were able to measure D-dimer 
A

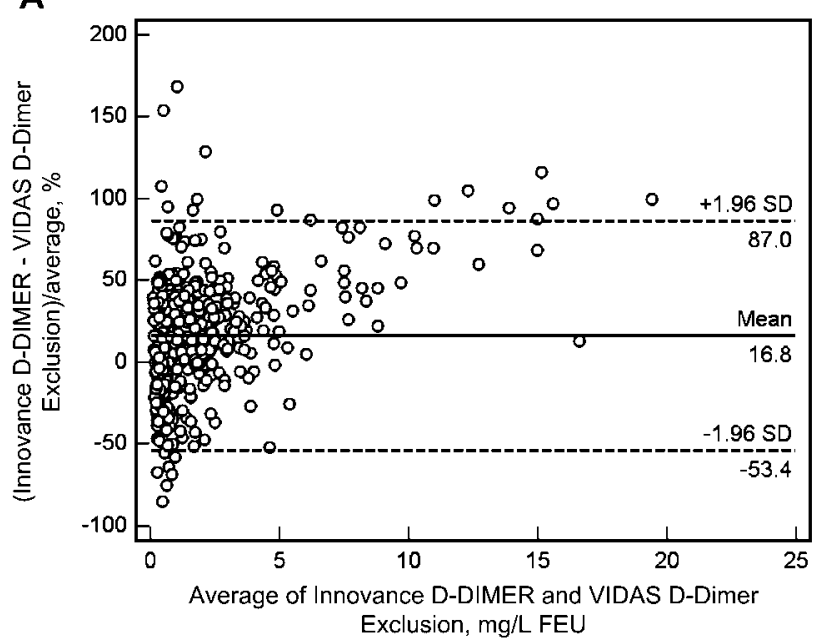

C

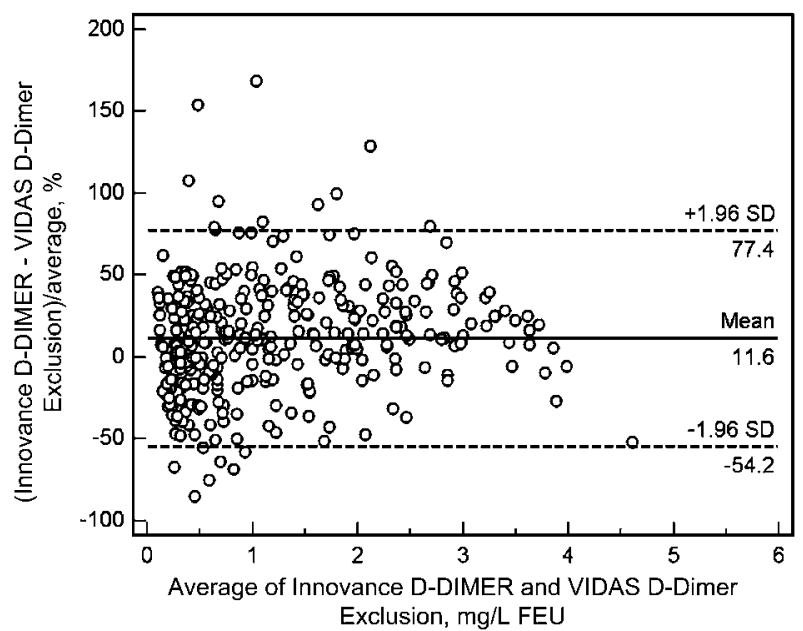

E

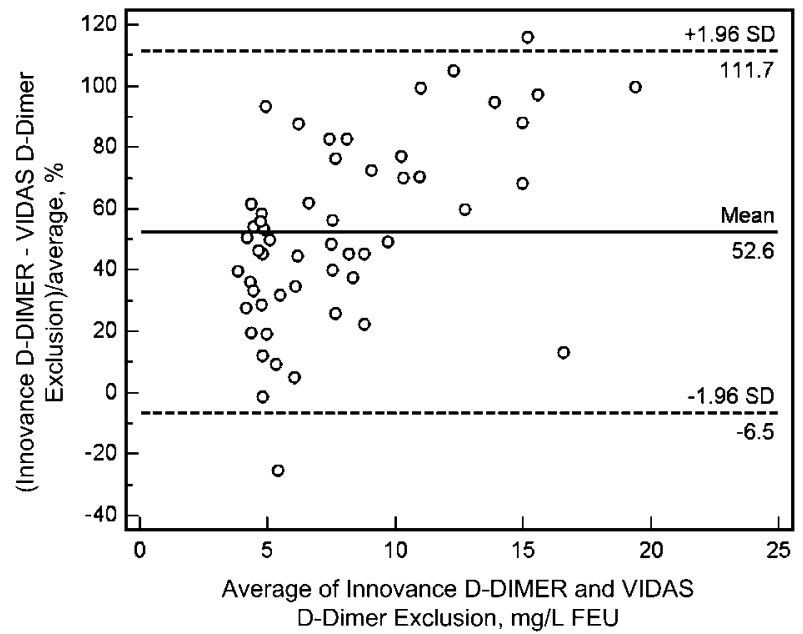

B

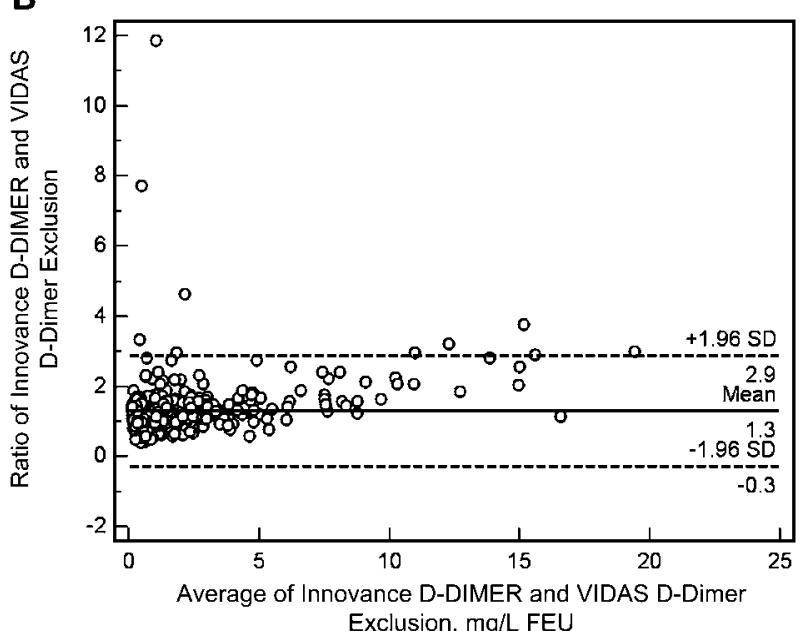

D

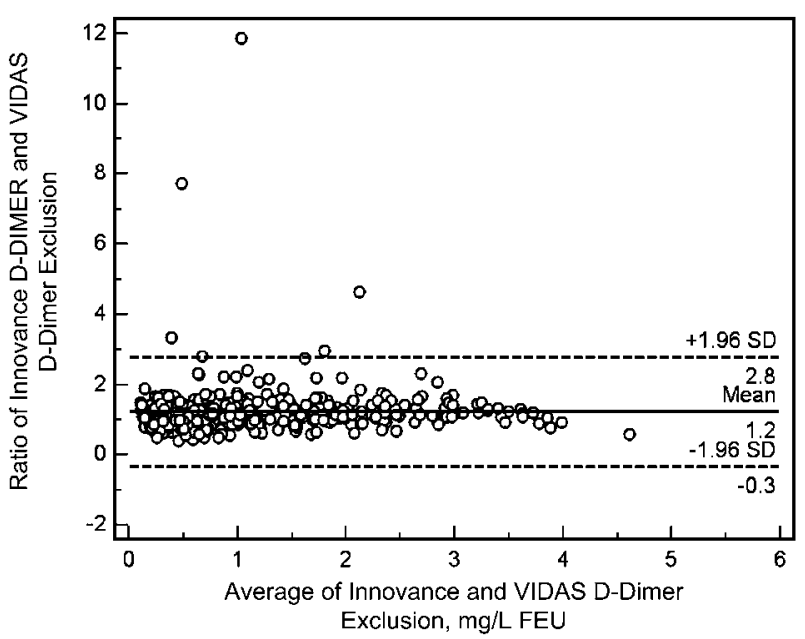

$\mathbf{F}$

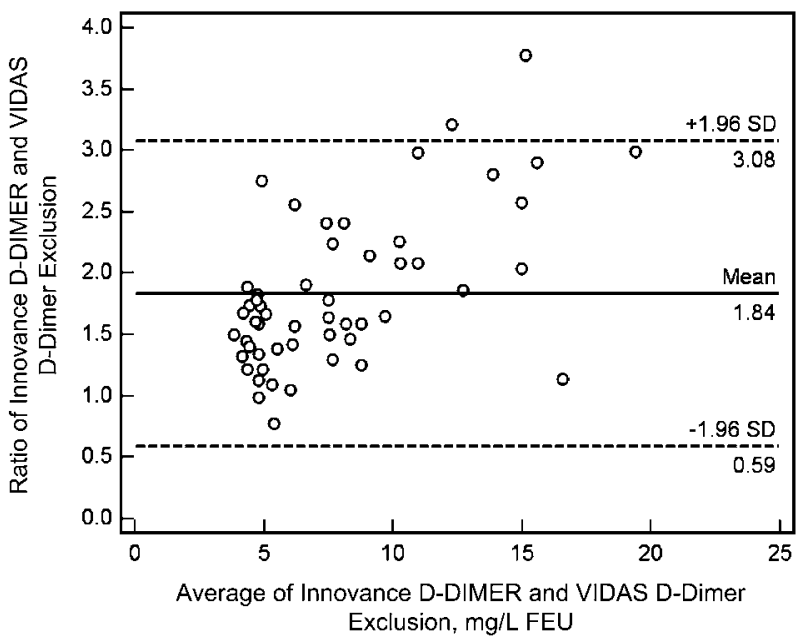

Figure 2 Method comparison between Innovance D-DIMER and VIDAS D-Dimer Exclusion according to Bland and Altman. Percent difference plot (A) and ratio difference plot (B) for all samples, percent difference plot (C) and ratio difference plot (D) for undiluted samples, percent difference plot $(E)$ and ratio difference plot $(F)$ for diluted samples.

concentrations that were below the lowest point of the calibration curve $(<0.17 \mathrm{mg} / \mathrm{L}$ FEU).

Method comparison studies were performed using the currently proposed D-dimer reference method, VIDAS D-Dimer Exclusion (10). In contrast to the majority of evaluation studies, we measured D-dimer using fresh plasma samples with both assays in order to exclude the potential effects of freezing on sample stability. In addition, the comparison of results that were obtained was simplified because results for both assays were expressed in the same units ( $\mathrm{mg} / \mathrm{L} \mathrm{FEU)}$ and the same recommended cut-off value $(<0.5$ $\mathrm{mg} / \mathrm{L}$ FEU) was used. Excellent correlation between Innovance D-Dimer assay and VIDAS D-Dimer Exclu- 
Table 3 Results of commercial D-Dimer control samples and calibration plasma samples obtained with D-Dimer assays.

\begin{tabular}{|c|c|c|c|c|}
\hline D-Dimer control/calibration plasma & $\begin{array}{l}\text { Lot } \\
\text { number }\end{array}$ & $\begin{array}{l}\text { Assigned value and } \\
\text { confidence interval, } \\
\mathrm{mg} / \mathrm{L} \mathrm{FEU}\end{array}$ & $\begin{array}{l}\text { VIDAS } \\
\text { D-Dimer } \\
\text { Exclusion, } \\
\text { mg/L FEU }\end{array}$ & $\begin{array}{l}\text { Innovance } \\
\text { D-DIMER, } \\
\text { mg/L FEU }\end{array}$ \\
\hline \multicolumn{5}{|l|}{ Control plasma } \\
\hline VIDAS C1 & $080509-0$ & $4.72(3.30-6.14)$ & 5.05 & 15.86 \\
\hline VIDAS C2 & $080509-0$ & $0.53(0.40-0.65)$ & 0.51 & 1.70 \\
\hline Innovance D-DIMER Control 1 & 560710 & $0.39(0.31-0.47)$ & 0.25 & 0.35 \\
\hline Innovance D-DIMER Control 2 & 560710 & $2.88(2.30-3.46)$ & 1.08 & 2.96 \\
\hline \multicolumn{5}{|l|}{ Calibration plasma } \\
\hline VIDAS S1 & 080509-0 & 4.63 & - & 15.18 \\
\hline VIDAS S2 & 080509-0 & 0.46 & - & 1.37 \\
\hline Innovance D-DIMER Calibrator & 560510 & 5.54 & 2.35 & - \\
\hline
\end{tabular}

FEU, fibrinogen equivalent units.

Table 4 Results of UK NEQAS plasma samples obtained with both D-Dimer assays.

\begin{tabular}{llll}
\hline $\begin{array}{l}\text { UK NEQAS } \\
\text { sample }\end{array}$ & $\begin{array}{l}\text { Innovance } \\
\text { D-DIMER, } \\
\text { mg/L FEU }\end{array}$ & $\begin{array}{l}\text { VIDAS D-Dimer } \\
\text { Exclusion, } \\
\text { mg/L FEU }\end{array}$ & $\begin{array}{l}\text { Ratio of Innovance } \\
\text { D-DIMER and VIDAS } \\
\text { D-Dimer Exclusion }\end{array}$ \\
\hline DD2006/1 & 3.69 & 2.44 & 1.51 \\
DD2006/2 & 1.93 & 1.40 & 1.38 \\
DD2006/3 & 1.13 & 0.78 & 1.45 \\
DD2006/4 & 1.49 & 1.49 & 1.00 \\
DD2006/5 & 1.32 & 1.08 & 1.22 \\
07/35 & 2.11 & 1.71 & 1.23 \\
\hline
\end{tabular}

UK NEQAS, UK National External Quality Assessment Scheme; FEU, fibrinogen equivalent units.

sion was obtained which was even better than the correlation obtained between both assays using the same analyzers described for a recent multicenter evaluation study by de Moerloose et al. (11). However, the results obtained in this study showed slightly higher results with Innovance D-DIMER that were more pronounced with increasing values as readily demonstrated by the Bland-Altman difference plots. Finally, as shown by the $\kappa$ coefficient in the method comparison study, very good agreement was found between the two methods.

Our results corroborate the previously published statement that a simple conversion factor between two assays cannot be applied to the entire concentration range since this can lead to discrepant results at both low and high measured values (1).

The 3-fold higher values obtained using VIDAS control samples measured with the Innovance D-DIMER is a consequence of the same difference observed when comparing assay-specific calibrators despite their being of the same origin (human). This is why manufacturers provide calibrators and control samples that are valid only for the assay for which they are designed (9).

For routine clinical use, assays for D-dimer must fulfill several criteria (12). This study shows that Innovance D-DIMER assay fulfills all the characteristics needed concerning ease of use and automated performance, short turnaround time of $10 \mathrm{~min}$, reliable measurement of values close to the cut-off value, assay availability of $24 \mathrm{~h}$ a day, even for single samples, and wide measurement range. In addition, the assay that was clinically validated in a recently pub- lished multicenter evaluation study by de Moerloose et al. (11) proved to be accurate when used for the diagnostic work-up of outpatients with venous thromboembolism.

\section{Statement}

We have not accepted any funding or support from any organization, we have not been employed by any organization that may in any way gain or lose financially from the results of our study, and we do not have any other conflicting interests.

\section{Acknowledgements}

We thank Siemens Medical Solutions Diagnostics for providing us with reagents.

\section{References}

1. Dempfle CE. D-dimer: standardization versus harmonization. Thromb Haemost 2006;95:399-400.

2. Dempfle CE. D-dimer assays: the current status and new assay technologies. Thromb Res 2006;118:569-71.

3. Clinical Laboratory and Standards Institute. Protocols for determination of limits of detection and limits of quantification; approved guidelines, CLSI document EP 17-A, Vol 24, No. 34. Wayne, PA: Clinical Laboratory and Standards Institute, 2004.

4. Pittet JL, de Moerloose P, Reber G, Durand C, Villard C, Piga N, et al. VIDAS D-dimer: fast quantitative ELISA for measuring D-dimer in plasma. Clin Chem 1996;42:410-5. 
5. Passing $H$, Bablok W. A new biometrical procedure for testing the equality of measurements from two different analytical methods. Application of linear regression procedures for method comparison studies in clinical chemistry, Part I. J Clin Chem Clin Biochem 1983;21:709-20.

6. Bland JM, Altman DG. Statistical methods for assessing agreement between two methods of clinical measurement. Lancet 1986;1:307-10.

7. Kramer MS, Feinstein AR. Clinical biostatistics. LIV. The biostatistics of concordance. Clin Pharmacol Ther 1981; 29:111-23.

8. Landis JR, Koch GG. The measurement of observer agreement for categorical data. Biometrics 1977;33:15974

9. Reber G, de Moerloose P. D-Dimer assays for the exclu- sion of venous thromboembolism. Sem Thromb Hemost 2000:26:619-24.

10. Guidelines on the diagnosis and management of acute pulmonary embolism: the Task Force for the Diagnosis and Management of Acute Pulmonary Embolism of the European Society of Cardiology (ESC). Eur Heart J 2008;29:2276-315.

11. de Moerloose P, Palareti G, Aguilar C, Legnani C, Reber G, Peetz D. A multicenter evaluation of a new quantitative highly sensitive D-dimer assay for exclusion of venous thromboembolism. Thromb Haemost 2008;100: 505-12.

12. Dempfle CE. Use of D-Dimer assays in the diagnosis of venous thrombosis. Sem Thromb Hemost 2000;26: 631-41. 